\title{
TAMAÑO DE ÁREA DE DRENAJE Y CONECTIVIDAD HIDROLÓGICA EN LA FORMACIÓN DE ESCORRENTÍA EN CUENCAS SEMIÁRIDAS MEDITERRÁNEAS. CUENCA AFORADA DEL BARRANC DEL CARRAIXET
}

\author{
J. A. Pascual Aguilar, J. L. Rubio, V. Andreu, J. Campo, O. González \& E. Gimeno \\ Centro de Investigaciones sobre Desertificación-CIDE (CSIC, UV, GV), Degradación y Conservación \\ de Suelos, Cami de la Marjal s/n, 46470 Albal (Valencia).E-mail: juan.a.pascual@uv.es
}

\begin{abstract}
The rainfall-runoff conversion is analysed taking in consideration the contributing area and hydrological connectivity, factors needing better understanding. Within the same basin, the Barranc del Carraixet near Valencia, has been selected three scales of work: four experimental plots ( $8 \times 40 \mathrm{~m})$, micro-basin $(17.01 \mathrm{ha})$ and small drainage basin $\left(127.9 \mathrm{~km}^{2}\right)$. For the analysis of runoff generation at different scales rainfall thresholds and runoff rates and thresholds were used. For hydrological connectivity, at plot level, coefficients of determination were analyzed. Inverse relationship is given between the volume of runoff and recurrence, with increasing scale. Connectivity, at plot level, is variable, increasing the $R^{2}$ according the simplification of processes, when the interference of vegetation decreases, establishing better linearity between rain and surface flow.
\end{abstract}

Keywords: Rainfall-runoff relationships; hydrological connectivity; drainage; contributing area; runoff generation.

RESUMEN.- Se analizan las relaciones de la conversión de la lluvia en escorrentía superficial atendiendo al tamaño del área de aporte y la conectividad hidrológica, factores poco estudiados. En una misma cuenca, la del Barranc de Carraixet en las cercanías de Valencia, se han seleccionado tres escalas de trabajo: cuatro parcelas experimentales $(8 \times 40 \mathrm{~m})$, microcuenca $(17,01 \mathrm{ha})$ y cuenca de drenaje de pequeño tamaño $\left(127,9 \mathrm{~km}^{2}\right)$. Para el análisis de la formación de escorrentía en las distintas escalas se utilizaron los umbrales de precipitación, indices y umbrales de escorrentía. Para la conectividad hidrológica, a escala de parcela, se analizaron los coeficientes de determinación. Se ha obtenido una relación inversa en volumen de escorrentía y en recurrencia, según aumenta la escala. La conectividad, a escala de parcela, es variable, aumentando el $R^{2}$ con la simplificación de los procesos, sobre todo al dis- 
J. A. PASCUAL AGUILAR, J. L. RUBiO, V. ANDREU, J. CAMPO, O. GONZÁLEZ \& E. GIMENO

minuir la interferencia de la vegetación, estableciéndose mayor linealidad entre la lluvia y el flujo superficial.

Palabras clave: Relaciones precipitación-escorrentía; conectividad hidrológica; drenaje; área de contribución; generación de escorrentía.

\section{Introducción}

La comprensión de los efectos de la escala y de interrelación de procesos que permiten la formación de escorrentía y su continuidad a lo largo del área de drenaje son retos importantes en el conocimiento de los sistemas hidrológicos de zonas de montaña mediterránea. Aunque, en general, existe una relación inversa entre el tamaño del área y la producción de escorrentía, se considera también que a distintas escalas existen unos procesos que dominan sobre otros. Por lo tanto, dependiendo de la escala, ya sea en el tiempo o en el espacio, los principios fundamentales o sus parámetros característicos tienen que ser modificados o interpretados de manera diferente para que, aun concurriendo los mismos procesos, pueda comprenderse el funcionamiento hidrológico adecuadamente (BRAUN et al., 1997). En grandes cuencas de drenaje los elementos más determinantes son el tamaño de la célula de precipitación, su intensidad y duración (YAIR \& RAZYASSIF, 2004). A escalas menores, la respuesta hidrológica sería más inmediata, dado que la importancia de los procesos no exclusivamente meteorológicos es mayor destacando la interrelación de zonas productoras (áreas fuente) y zonas receptoras (áreas sumidero) de manera que, en condiciones favorables, produzcan suficiente escorrentía superficial que alcancen a producir caudal. En este caso, la estructura parcheada de la superficie es determinante, actuando las áreas vegetadas como sumideros, o áreas de re-infiltración de la escorrentía, en contraste con las zonas sin vegetación que funcionan como áreas de contribución o generación de escorrentía (IMESON \& PRINSEN, 2004).

En la actualidad se utiliza el término de conectividad hidrológica para establecer las conexiones entre los procesos que gobiernan la producción de escorrentía, independientemente de la escala de análisis, y su continuidad superficial, al reconocerse la necesidad de abundar en el conocimiento sobre los procesos de generación de escorrentía, más allá de las ideas tradicionales sobre la generación de flujos (BRACKEN \& CROKE, 2007).

La conectividad (por medio de la variabilidad) y los efectos de la escala en la generación de escorrentía (o procesos hidrológicos) han sido analizadas de 
manera indirecta con modelos hidrológicos distribuidos de base física, tanto comparando datos reales con las estimaciones obtenidas en el modelo (YILDIZ \& BARROS, 2009) como por medio de la creación de escenarios de simulación (SHA et al., 1996). Asímismo, el enfoque empírico de detalle para esclarecer el comportamiento de las estructuras en mosaico de zonas con y sin vegetación también ha sido utilizado (CAMMERAAT \& IMESON, 1999; LUDWIG et al., 2000). Sin embargo, son escasos los trabajos que plantean una metodología escalar, basada en recogida de información directa, donde se establezcan criterios de análisis a distintas escalas de trabajo dentro de una misma cuenca de drenaje.

El objetivo de este trabajo es estudiar las características de conectividad y la influencia de la escala en la formación de escorrentía superficial en tres unidades hidrológicas de distinto tamaño. Para ello se han seleccionado dentro de una misma unidad, bajo el concepto de cuencas anilladas, tres zonas de trabajo distintas que representarían adecuadamente tres escalas distintas de trabajo. Estas son las de parcela experimental, la microcuenca de drenaje de pocas hectáreas y la cuenca de drenaje de medianas dimensiones de algo más de $127,9 \mathrm{~km}^{2}$ de superficie.

\section{2. Área de estudio}

Los tres ámbitos de estudio (cuenca aforada del Carraixet, microcuenca y parcelas experimentales (P1, P2, P3 y P4, respectivamente) forman parte de una misma cuenca de drenaje, el Barranc de Carraixet (Figura 1).

En el contexto general de la cuenca aforada del Carraixet $\left(127,9 \mathrm{~km}^{2}\right)$, el tipo climático es seco subhúmedo, con moderado exceso de agua en invierno. Pertenece al piso Termomediterráneo (media anual $15^{\circ} \mathrm{C}$ ) y presenta un ombroclima seco (precipitación anual $572 \mathrm{~mm}$ ). La litología está caracterizada de norte a sur, por materiales triásicos del Muschelkalk (constituidos por margas arcilloso-areniscosas, areniscas, dolomías y conglomerados), calizas jurásicas y materiales más recientes de depósitos cuaternarios. Son frecuentes los afloramientos de material fuertemente consolidado. Los suelos, dependiendo del material de origen y su posición topográfica, se incluyen en Regosoles, Leptosoles, Fluvisoles, Cambisoles y Calcisoles (FAO-UNESCO, 1988). Florísticamente, la zona se encuadra en el ámbito geográfico de la serie termo-mesomediterránea ibero-levantina basófila de la carrasca (COSTA, 1999), donde las especies definitorias son la carrasca (Quercus rotundifoliae), el palmito (Chamaerops humilis), el bayón o ginestell (Osyris quadripartita), la rubia de hoja larga (Rubia peregrina subsp. Longifolia). 


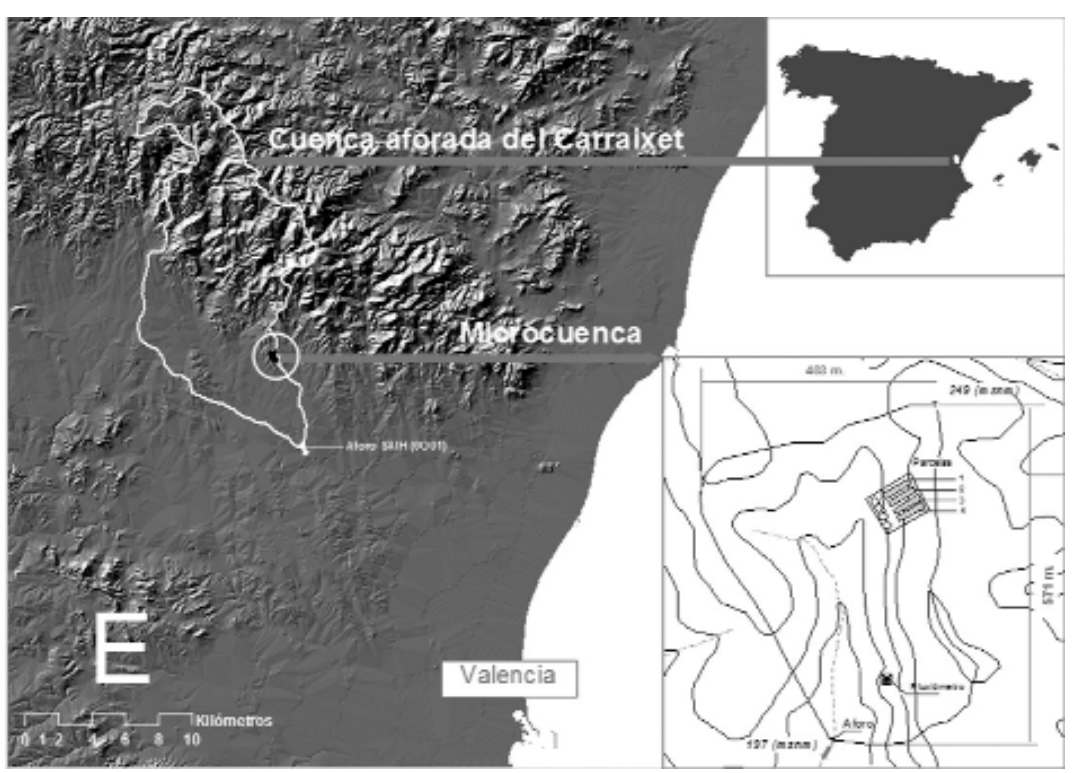

Figura 1. Localización e instrumentalización del área de estudio. Figure 1. Location of the study area and instrument setting.

Debido a las sucesivas intervenciones del hombre se han producido importantes modificaciones tanto en la composición de la vegetación como en los usos y cubiertas del suelo, estando ocupada su superficie en la actualidad por un 6,5\% de cubiertas artificiales (edificaciones, y vías de comunicación), un 20,7 por cultivos de regadío (mayoritariamente cítricos), un 16,9\% por cultivos de secano arbolados (almendro, olivo y algarrobo) y un $60 \%$ de vegetación natural, cuya composición y estructura es variable a tenor de la incidencia de los incendios naturales y de las distintas repoblaciones. Entre estas últimas, destacan las realizadas a mediados del siglo pasado con Pinus halepensis y Pinus pinaster.

La microcuenca $(17,01 \mathrm{ha})$, con un rango altudinal de 52 metros, es tributaria del Barranc del Carraixet en su sector central. Debido a su menor tamaño posee características ambientales más homogéneas. La litología está caracterizada por materiales triásicos del Muschelkalk constituidos por margas arcilloso-areniscosas y conglomerados que, por su fácil meteorización y fragmentación, dan lugar a abundante pedregosidad. Son frecuentes los afloramientos de material fuertemente consolidado. El suelo dominante se ha clasificado como Leptosol réndsico (FAO-UNESCO, 1988), de espesor 
variable pero escaso. Su textura es franco-arcillo-arenosa, con un $\mathrm{pH}$ de $\sim 8.0$ y un contenido de materia orgánica $\sim 5.6 \%$.

En superficie se da una combinación florística en forma de parches en los que se mezclan en distintas proporciones los afloramientos rocosos y suelo desnudo, la vegetación de poco porte y estructuras de vegetación de mayor porte e incluso algunas manchas arboladas con pino de repoblación (Tabla 1). Las especies dominantes son Quercus coccifera, Pistacia lentiscus, Rosmarinus officinalis, Erica multiflora, Stipa tenacissima, Globularia alypum y Thymus vulgaris.

Tabla 1. Características de la vegetación/cubierta del suelo de la microcuenca. Table 1. Vegetation/soil cover characteristics in the micro-basin.

\begin{tabular}{|lcc|}
\hline \multicolumn{1}{|c|}{ Tipo de cubierta } & Superficie $\left(\mathrm{m}^{2}\right)$ & $\%$ \\
\hline Rocas aflorantes/Suelos desnudo & 39741.6 & 23.3 \\
Vegetacion herbácea-arbustiva de poco porte & 102509.6 & 60.1 \\
Vegetacion arbustiva alta-arbolado & 28313.8 & 16.6 \\
Total & 170565.0 & \\
\hline
\end{tabular}

Las 4 parcelas experimentales se encuentran localizadas dentro de la microcuenca en su parte más alta noroccidental. Tienen una superficie de 320 $\mathrm{m}^{2}(8 \times 40 \mathrm{~m})$ y condiciones ambientales iguales. Inicialmente, en 1996 fueron sometidas a tratamiento de incendios experimentales a excepción de las parcelas 1 y 3, en la que se mantuvo la estructura de vegetación original. Desde entonces la vegetación ha consolidado su proceso de recuperación, existiendo algunas diferencias en la composición florística y en el grado de cubierta del suelo (Tabla 2), siendo la Parcela 2 la que mayor porcentaje de suelo desnudo y afloramientos rocosos presenta.

\section{Metodología}

\subsection{Instrumentación y recogida de datos}

Cuatro tipos de datos provenientes de dos distintas fuentes de información han sido utilizados en este trabajo. La escorrentía producida en las parcelas, la escorrentía aforada de la microcuenca y las series de precipitación forman parte de la instrumentalización de la microcuenca 
J. A. PASCUAL AGUILAR, J. L. RUBIO, V. ANDREU, J. CAMPO, O. GONZÁLEZ \& E. GIMENO

Tabla 2. Características de la vegetación y cubierta superficial en las parcelas.

Table 2. Vegetation and soil cover characteristics in the experimental plots.

\begin{tabular}{|c|c|c|c|c|}
\hline & PARCELA 1 & PARCELA 2 & PARCELA 3 & PARCELA 4 \\
\hline $\begin{array}{l}\% \text { Cubierta } \\
\text { Vegetal }\end{array}$ & 55 & 50 & 55 & 60 \\
\hline $\begin{array}{l}\text { \% suelo } \\
\text { desnudo }\end{array}$ & $\begin{array}{c}32+5 \% \text { de musgos } \\
\text { tapizando el suelo }\end{array}$ & 40 & 37 & 30 \\
\hline $\begin{array}{l}\% \text { afloramientos } \\
\text { rocosos }\end{array}$ & 8 & 10 & 8 & 10 \\
\hline $\begin{array}{l}\text { Especies } \\
\text { principales } \\
\text { por orden de } \\
\text { dominancia }\end{array}$ & $\begin{array}{l}\text { Rosmarinus officinalis, } \\
\text { Anthyllis cytisoides, } \\
\text { Globularia alypum, } \\
\text { Stipa tenacisima, } \\
\text { Brachypodium retusum }\end{array}$ & $\begin{array}{l}\text { Brachypodium retusum, } \\
\text { Anthyllis cytisoides, } \\
\text { Rosmarinus officinalis, } \\
\text { Globularia alypum, } \\
\text { Thymus vulgaris }\end{array}$ & $\begin{array}{c}\text { Rosmarinus officinalis, } \\
\text { Stipa tenacísima, } \\
\text { Globularia alypum, } \\
\text { Pistacia lentiscos, } \\
\text { Anthyllis cytisoides }\end{array}$ & $\begin{array}{l}\text { Stipa tenacissima, } \\
\text { Rosmarimus officinalis, } \\
\text { Globularia alypum, } \\
\text { Juniperus oxycedrus, } \\
\text { Quercus coccifera, }\end{array}$ \\
\hline Presencia & Thymus vulgaris, & Chamaerops humilis & $\begin{array}{l}\text { Erica multiflora, } \\
\text { Thymus vulgaris }\end{array}$ & $\begin{array}{c}\text { Rhamnus lycioides } \\
\text { Pistacia lentiscus, } \\
\text { Erica multiflora, } \\
\text { Pinus halepensis (1 } \\
\text { ejemplar de } 170 \mathrm{~cm} \\
\text { de altura) }\end{array}$ \\
\hline
\end{tabular}

(Figura 1). Para los caudales de la cuenca aforada del Carraixet se han analizado la información de la Red SAIH (Sistema Automático de Información Hidrológica en la Confederación Hidrográfica del Júcar) suministrada por el Ministerio de Medio Ambiente.

Aunque en este trabajo sólo se estudia la escorrentía, las parcelas (P1, P2, P3 y P4) están construidas de manera que recojan tanto la escorrentía como los sedimentos producidos. Tienen unas dimensiones de $40 \times 8 \mathrm{~m}$, con su lado mayor perpendicular a las curvas de nivel. Están delimitadas con ladrillos de $30 \times 50 \mathrm{~cm}$. En la parte inferior se estrechan para lograr la confluencia de los flujos, reduciendo su amplitud a $2 \mathrm{~m}$. Estos flujos se recogen en un colector de $2 \mathrm{~m}$ de frente, que a su vez se conecta mediante un tubo de PVC con un depósito de $2.000 \mathrm{~L}$ de capacidad, dentro del cual se coloca otro segundo depósito más pequeño de $30 \mathrm{~L}$, que permite recoger la escorrentía de sucesos de baja intensidad.

La medición de la escorrentía de la microcuenca se realiza mediante un aforo de pequeñas dimensiones localizado en el punto de confluencia de tres subunidades con pendientes relativamente similares pero orientación distinta. El medidor, RBC Flume de Eijkelkamp®, es de tipo trapezoidal de $1,5 \mathrm{~m}$ de largo por $1 \mathrm{~m}$ de ancho construido en acero inoxidable y apropiado para caudales de superficies muy pequeñas. Por medio de un mecanismo de presión, mide la altura de la lámina de agua en milímetros. El medidor de 
presión está conectado a un sistema digital de almacenamiento (data logger) de ocho canales. Está alimentado con una célula fotovoltaica y programado para que recoja escorrentía, cuando se produce, cada 15 segundos.

La precipitación se recoge por medio de un pluviómetro con un sistema de cuchara simple de balancín autodescargable, modelo ECRN-50 de DECAGON. Es un instrumento de pequeñas dimensiones $(5 \times 10 \mathrm{~cm})$ de precisión de $1 \mathrm{~mm}$ que se ha calibrado para que mida la lluvia a intervalos de 60 segundos. Está conectado a un data logger de seis canales, alimentado con baterías y con capacidad máxima de almacenamiento de registros (con la resolución temporal de 60 segundos) de 24 días.

La información requerida para el análisis de la escorrentía a escala de cuenca pertenece al Aforo SAIH 0O01, denominado Carraixet. Se trata de un sistema totalmente automatizado que recoge lecturas de la altura de la lámina de agua (a modo de limnímetro), siendo almacenada de manera digital en un data logger y enviada en tiempo real (vía satélite o radio) a las estaciones de control (MMAMRM, 2008). La información se almacena con una resolución temporal de 5 minutos, aunque los datos disponibles de libre acceso tienen un máximo detalle de 15 minutos.

\subsection{Tratamiento preliminar de la información}

Debido a que la instrumentalización de la microcuenca con el pluviómetro y el aforo de pequeñas dimensiones se realizó a principios de marzo de 2008, la información recogida para el análisis de este trabajo corresponde al primer año de funcionamiento de dichos aparatos, cubriendo desde el 1 de abril de 2008 hasta el 31 de marzo de 2009.

Previo al análisis, los datos se organizaron para que pudieran ser integrados de manera homogénea en las distintas comparaciones establecidas. Por lo tanto, el tratamiento inicial al que se sometieron dependió tanto de la forma de recogida como de la dimensión temporal de almacenamiento de los instrumentos. Los datos de precipitación fueron agrupados en lluvias acumuladas en 24 horas y agrupados en series (o tandas) de precipitación, considerando que para una misma tanda no debían superarse las 24 horas sin precipitación. Los datos de caudal, una vez analizados los registros del aforo de la microcuenca y SAIH, se agruparon por el período de escorrentía, obteniéndose un valor total de caudal en litros, realizándose los cálculos necesarios para convertir en todos los casos las unidades de medida originales ( $\mathrm{mm}$ de altura del pluviómetro $\mathrm{y}^{3} / \mathrm{s}$ en el Aforo SAIH) a litros totales. 


\subsection{Análisis}

Se han establecido dos tipos de análisis, por un lado la producción de escorrentía de acuerdo a la escala de las áreas de drenaje y, por otro, la conectividad hídrica a escala de parcela y su relación con los volúmenes de escorrentía debido a distintos tipos de cubiertas superficiales.

Para el primer caso se realizó, en primer lugar, un análisis comparativo entre el número de sucesos de lluvia acumulada en 24 horas agrupados por tandas y las respectivas respuestas a escala de parcela, microcuenca y cuenca. En segundo lugar, se obtuvieron porcentajes y tasas de escorrentía. El porcentaje de escorrentía (PE) es, en realidad, una adaptación del índice de escorrentía, entendido como la fracción de agua total $\left(E_{t}\right)$ después de un suceso referida al volumen total de lluvia precipitada $\left(\mathrm{P}_{\mathrm{t}}\right)$ sobre la misma superficie drenante: $P E=E_{t} / P_{t}{ }^{*} 100$. Por tasa de escorrentía (TE) se entiende la cantidad de escorrrentía total $\left(\mathrm{E}_{\mathrm{t}}\right)$ de un suceso determinado dividida por la superficie del área drenante $(\mathrm{A}): \mathrm{TE}=\mathrm{E}_{\mathrm{t}} / \mathrm{A}$.

En el caso de las parcelas, se ha realizado un análisis de correlación entre la lluvia y la escorrentía. La correlación empleada, atendiendo a que sólo se consideran dos variables, ha sido la regresión lineal con obtención de la función de ajuste a la recta y establecimiento del coeficiente de determinación $\left(\mathrm{R}^{2}\right)$.

\section{Resultados y discusión}

\subsection{Tamaño del área de aporte y producción de escorrentía}

En ambientes mediterráneos, un factor importante a destacar es la generación de escorrentía, donde la escala se demuestra, y a tenor de los resultados obtenidos, fundamental. Al tamaño de parcela experimental, la respuesta hidrológica es rápida, produciéndose flujo superficial en las cuatro parcelas estudiadas en más de la mitad de los sucesos de lluvia registrados. Sin embargo, su respuesta hídrica, es variable con respecto a la precipitación total. Si se comparan las respuestas de las parcelas con sus umbrales de escorrentía (Tabla 3), entendidos como volumen de precipitación mínimo necesario para que se produzca excedente de agua en la superficie del suelo, oscilan entre 1 y 9 milímetros (sucesos S-23, S-10 y S-24). Siempre para lluvias registradas en 24 horas. 
Tabla 3. Registro de sucesos de precipitación con las respectivas respuestas de escorrentía para las parcelas experimentales (P1, P2, P3 y P4), la microcuenca (MC) y la cuenca aforada del Carraixet (CAR) desde abril de 2008 a marzo de 2009.

Table 3. Rainfall and Overland flow registers for the experimental plots (P1, P2, P3 and P4), the microbasin and the Carraixet basin from April 2008 and March 2009.

\begin{tabular}{|c|c|c|c|c|c|c|c|c|c|c|}
\hline \multirow[t]{2}{*}{ Suceso } & \multicolumn{2}{|c|}{ Fecha } & \multirow[t]{2}{*}{$P(\mathrm{~mm})$} & \multirow[t]{2}{*}{ Días } & \multicolumn{4}{|c|}{ Parcelas } & \multirow[t]{2}{*}{$M C$} & \multirow[t]{2}{*}{$C A R$} \\
\hline & & & & & P1 & P2 & P3 & $P 4$ & & \\
\hline S-1 & $09 / 04 / 2008$ & $09 / 04 / 2008$ & 3.0 & 1 & 一 & - & 一 & - & - & 一 \\
\hline S-2 & $20 / 04 / 2008$ & $20 / 04 / 2008$ & 5.0 & 1 & 一 & - & 一- & 一 & - & 一 \\
\hline S-3 & $08 / 05 / 2008$ & $11 / 05 / 2008$ & 49.0 & 4 & $X$ & $X$ & $x$ & $X$ & - & 一 \\
\hline S-4 & $14 / 05 / 2008$ & $15 / 05 / 2008$ & 2.0 & 2 & 一 & - & 一 & 一 & - & 一 \\
\hline S-5 & $27 / 05 / 2008$ & $28 / 05 / 2008$ & 7.0 & 2 & 一 & - & 一 & 一 & - & 一 \\
\hline S-6 & $04 / 06 / 2008$ & $05 / 06 / 2008$ & 14.0 & 2 & $X$ & $X$ & $X$ & $X$ & - & 一 \\
\hline S-7 & $08 / 06 / 2008$ & $10 / 06 / 2008$ & 43.0 & 3 & 一 & - & 一 & 一 & - & 一 \\
\hline S-8 & $03 / 07 / 2008$ & $03 / 07 / 2008$ & 2.0 & 1 & 一 & - & 一 & 一 & - & 一 \\
\hline S-9 & $21 / 09 / 2008$ & $24 / 09 / 2008$ & 26.0 & 4 & $X$ & $X$ & $x$ & $X$ & - & 一 \\
\hline S-10 & $28 / 09 / 2008$ & $28 / 09 / 2008$ & 4.0 & 1 & $x$ & $X$ & $x$ & $x$ & 一 & 一 \\
\hline S-11 & $09 / 10 / 2008$ & $13 / 10 / 2008$ & 86.0 & 5 & $x$ & $X$ & $x$ & $x$ & $x$ & 一 \\
\hline S-12 & $18 / 10 / 2008$ & $19 / 10 / 2008$ & 11.0 & 2 & $x$ & $X$ & $x$ & $x$ & 一 & 一 \\
\hline S-13 & $22 / 10 / 2008$ & $24 / 10 / 2008$ & 19.0 & 3 & 一 & - & 一 & 一 & - & 一 \\
\hline S-14 & $28 / 10 / 2008$ & $29 / 10 / 2008$ & 33.0 & 2 & $x$ & $X$ & $x$ & $x$ & $x$ & 一 \\
\hline S-15 & $31 / 10 / 2008$ & $02 / 11 / 2008$ & 44.0 & 3 & $x$ & $X$ & $x$ & $x$ & $X$ & 一 \\
\hline S-16 & $12 / 11 / 2008$ & $12 / 11 / 2008$ & 1.0 & 1 & 一 & 一 & 一 & 一 & - & 一 \\
\hline S-17 & $08 / 12 / 2008$ & $10 / 12 / 2008$ & 22.0 & 3 & $X$ & $X$ & 一 & $X$ & - & 一 \\
\hline S-18 & $28 / 12 / 2008$ & $29 / 12 / 2008$ & 2.0 & 2 & 一 & - & 一 & - & - & 一 \\
\hline S-19 & $01 / 01 / 2009$ & $01 / 01 / 2009$ & 1.0 & 1 & 一 & 一 & 一 & 一 & - & 一 \\
\hline $\mathrm{S}-20$ & $03 / 01 / 2009$ & $05 / 01 / 2009$ & 24.0 & 3 & $X$ & $x$ & $X$ & $X$ & - & 一- \\
\hline S-21 & $31 / 01 / 2009$ & $02 / 02 / 2009$ & 26.0 & 3 & $X$ & $X$ & $x$ & $X$ & - & 一 \\
\hline S-22 & $15 / 02 / 2009$ & $15 / 02 / 2009$ & 2.0 & 1 & - & - & - & - & - & 一 \\
\hline S-23 & $21 / 02 / 2009$ & $21 / 02 / 2009$ & 1.0 & 1 & $X$ & $X$ & $x$ & $x$ & - & 一 \\
\hline S-24 & $28 / 02 / 2009$ & $28 / 02 / 2009$ & 9.0 & 1 & $x$ & $X$ & $x$ & $x$ & - & 一 \\
\hline S-25 & $03 / 03 / 2009$ & $03 / 03 / 2009$ & 15.0 & 1 & $x$ & $x$ & $x$ & $x$ & - & - \\
\hline S-26 & $28 / 03 / 2009$ & $31 / 03 / 2009$ & 54.0 & 4 & $x$ & $X$ & $x$ & $x$ & - & 一- \\
\hline
\end{tabular}

En la producción de escorrentía a escala de parcela, la conectividad, y por lo tanto la creación de excedentes hídricos, depende tanto de la cantidad de agua precipitada y de su intensidad, como de la duración y continuidad en el tiempo. Factores que se ven reflejados en la disminución de sucesos sin escorrentía a medida que se incrementa el número de días en las tandas de lluvia. La variabilidad en la respuesta también estaría relacionada con las condiciones antecedentes de humedad siendo más errática y dispersa con contenidos de humedad bajos y más lineal ante contenidos altos, como sugieren SHA et al. (1996).

Cuando se produce un volumen mayor de lluvia y condiciones de exfiltración favorables (reflejado en contenidos de humedad altos en el suelo, provenientes de lluvias antecedentes), es cuando a escala de microcuenca el 
sistema hídrico funciona, registrándose aportes en el caudalímetro. Su incidencia, o recurrencia, es considerablemente menor que a la escala de parcela. En este caso, al existir mayor diversidad, además de topográfica, de las características de la cubierta vegetal, el incremento de la complejidad del sistema hídrico, traducido en una mayor interacción de las áreas (fuente productoras de escorrentía) y sumidero (infiltradoras) conducirían a la disminución de la conectividad hídrica y, en última instancia a la creación de un caudal en el punto de aforo como síntesis de la misma.

A escala de microcuenca cabría introducir también un elemento, el del tamaño de la célula de precipitación, pues posiblemente se requerirá una mayor extensión del área de precipitación sobre la superficie de la cuenca. Sin embargo, dichos mecanismos, aunque necesarios por si solos, no son suficientes para iniciar una respuesta hidrológica a escala de cuenca con varios kilómetros cuadrados de área de aporte, por lo que habría que considerar, además de la mayor complejidad de la misma, tanto en sus componentes topográficos como ambientales, la importancia de la escala o tamaño del suceso meteorológico. Ene este último, posiblemente deban coincidir también el tamaño de la célula de precipitación (YAIR \& RAZYASSIF, 2004) y la propia dinámica y recorrido de las masas nubosas (CAMARASA, 1995), además de su intensidad.

Para la cuenca del Carraixet, los registros existentes desde el momento de la instalación son escasos, habiéndose registrado cuatro eventos desde la instalación del aforo en 1986. En estos casos, un factor importante que corrobora la idea de que a la escala de cuenca son necesarias precipitaciones totales por unidad de superficie mayores y sucesos que alcancen toda su superficie, son las dimensiones de las mismas. En todas las estaciones de referencia analizadas para caracterizar la naturaleza de la precipitación de los sucesos (CAMARASA, 1995; OLIVARES GUILLEM, 2004) los totales superan en dos casos los $100 \mathrm{~mm}$. Situación muy semejante en cuencas vecinas con características no totalmente coincidentes a las de la cuenca del Carraixet (CAMARASA, 1990).

Escala y conectividad van íntimamente interrelacionadas como se observa tanto en los índices (o porcentajes) como en las tasas de escorrentía (Tabla 4). El primero relaciona la escorrentía con la magnitud de la lluvia, mientras que el segundo la relaciona con la superficie drenante o la capacidad exfiltrante de los suelos. Tanto en las parcelas como en la microcuenca, los porcentajes de escorrentía son bajos, siendo en la mayoría de registros muy inferiores a 2 . Es en las parcelas donde los valores son más dispares y extremos alcanzándose porcentaje máximos de escorrentía de 20,9 (S.23) en P4, 11,3 en P2, 9,8 en P1 y 5,0 en P3. Por el contrario, la escorrentía registrada en la microcuenca define un comportamiento menos dispar siendo los porcentajes 
inferiores al 0,5 del volumen de agua total precipitada. Las tasas de escorrentía por unidad de superficie corroboran esa menor capacidad productiva de la microcuenca, pues en los tres casos registrados los valores están en 0,1 .

\subsection{Composición florística y conectividad hidrológica a la escala de ladera}

Por medio de los coeficientes de determinación (Figura 2) se establece una relación entre la precipitación y la escorrentía producida en cada parcela experimental. Se asume en el análisis que el coeficiente de determinación obtenido refleja la influencia del grado y tipo de cubierta vegetal. Aunque en las cuatro parcelas analizadas las correlaciones entre precipitación y escorrentía son dispares. Pueden destacarse comportamientos en alguna parcela que corroboran una mayor conectividad hidrológica. La mejor determinación ocurre en P2 $\left(R^{2}=0,55\right)$ y, en menor medida, en P1 $\left(R^{2}=0,11\right)$. En la primera cabe destacar el alto porcentaje de suelo desnudo y afloramientos rocosos existente, alcanzando en conjunto el $50 \%$ de la superficie total (Tabla 2), por lo que la interrelación existente entre áreas generadoras de escorrentía (sin vegetación) y almacenadoras de agua (con vegetación) que sugiere CAMMERAAT (2004) quedaría debilitada, creando un sistema más lineal, donde los principales componentes del régimen hídrico serían la precipitación y la escorrentía con disminución de los procesos intermedios de la vegetación. Por lo tanto, ante condiciones pluviométricas idénticas, las parcelas tendrán un comportamiento distinto debido a si la superficie es afloramiento rocoso, suelo desnudo o suelo cubierto por la vegetación y, además, cuando se da esta última posibilidad, de la estructura de la biomasa y composición florística. Lógicamente, el coeficiente será más determinante entre las variables analizadas a medida que se simplifican las condiciones ambientales.

\section{Conclusiones}

Se observan diferencias apreciables en la generación de escorrentía en las tres escalas analizadas. La respuesta en las parcelas es rápida ocurriendo en una alta proporción de los eventos de precipitación. A medida que se aumenta el área drenante, la respuesta, en cuanto a la formación de flujos de síntesis recogidos como caudal, es menor, reduciéndose considerablemente el número de sucesos de escorrentía a escala de microcuenca, y nula -durante el año analizado- a la escala de cuenca de drenaje. En la respuesta de las tres 

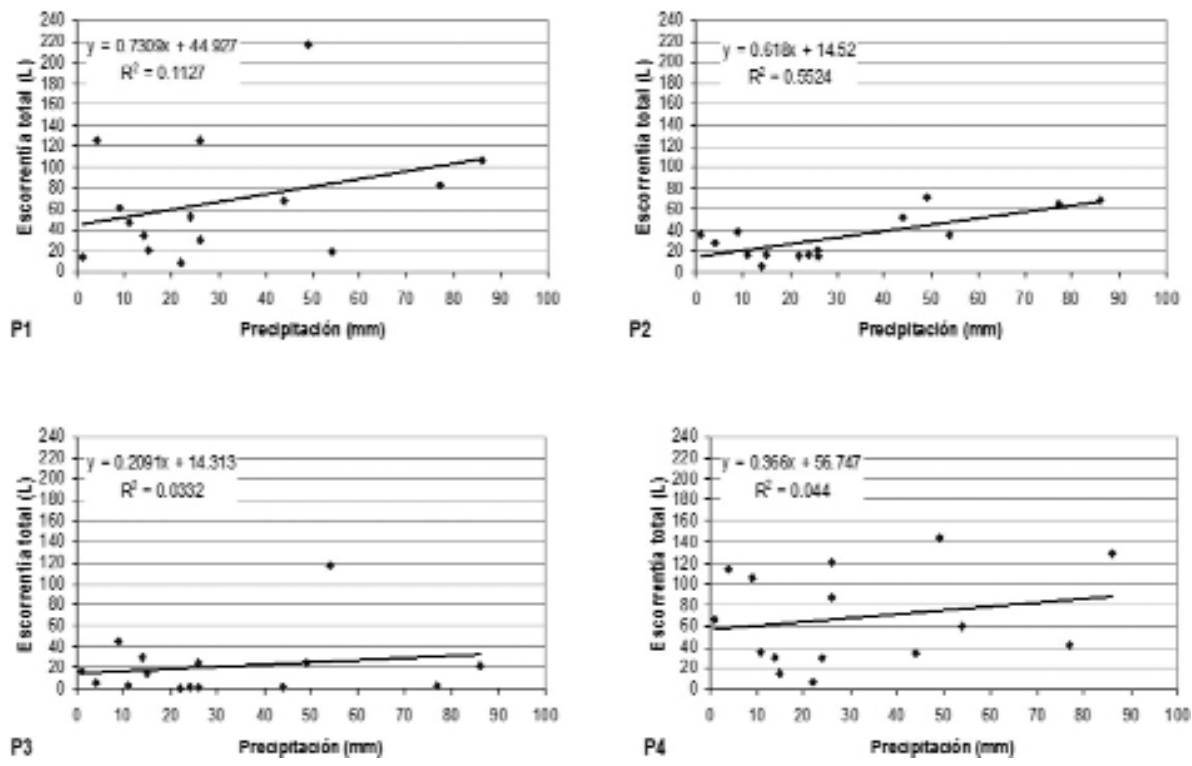

Figura 2. Coeficientes de determinación entre precipitación y escorrentía de las parcelas experimentales.

Figure 2. Regression coefficients between rainfall and overland flow for the experimental plots.

escalas analizadas también hay que considerar los umbrales mínimos de precipitación necesarios, los cuales se incrementan con el tamaño del área drenante, e irán estrechamente asociados al tamaño y la intensidad de la célula de precipitación.

En un alto porcentaje de los sucesos de respuesta la fracción de agua precipitada recogida como escorrentía es baja, por lo que, además de las retenciones producidas por la vegetación cabría pensar en un sistema de pérdidas edafo-litológico con gran importancia de la infiltración y de almacenamientos superficiales que limitan la conectividad hidrológica en superficie. La vegetación juega un papel importante en dicha conectividad al matizar la relación directa entre la lluvia y la escorrentía, haciendo las interrelaciones del sistema más complejas, como se observa en los coeficientes de correlación más altos de las parcelas donde se da un porcentaje alto de suelo sin vegetación. 


\section{Referencias}

BRACKEN, L.J. \& CROKE, J. 2007. The concept of hydrological connectivity and its contribution to understanding runoff-dominated geomorphic systems. Hydrological Processes, 21: 1749-1763.

BRAUN, P.; MOLNAR, T. \& KLEEBERG, H.B., 1997. The problem of scaling in grid-related hydrological process modeling. Hydrological Processes, 11: 1219-1230.

CAMARASA, A.M., 1990. Génesis de avenidas en pequeñas cuencas semiáridas: la Rambla de Poyo (Valencia). Cuadernos de Geografía, 48: 81104.

CAMARASA, A.M., 1995. Génesis de crecidas en pequeñas cuencas semiáridas: Barranc de Carraixet y Rambla de Poyo. Ministerio de Obras Públicas, Transporte y Medio Ambiente, 252 pp., Madrid.

CAMMERAAT, E.L.H., 2004. Scale dependent thresholds in hydrology and erosion response of a semi-arid catchment in south-east Spain. Agriculture, Ecosystems and Environment, 104: 317-332.

CAMMERAAT, E.L.H. \& IMESON, A.C., 1999. The significance of soilvegetation patterns following land abandonment and fire in Spain. Catena, 37: 107-127.

COSTA, M., 1999. La vegetación y el paisaje en las tierras valencianas. Rueda, 342 pp., Madrid.

FAO-UNESCO, 1988. Soil map of the world. Revised legend 1:5.000.000. FAO, 119 pp., Roma.

IMESON, A.C. \& PRINSEN, H.A.M., 2004. Vegetation patterns as biological indicators for identifying runoff and sediment source and sink areas for semi-arid landscapes in Spain. Agriculture, Ecosystems and Environment, 104: 333-342.

LUDWIG, J.A.; WIENS, J. \& TONGWAY, D.J., 2000. A scaling rule for landscape patches and how it applies to conserving soil resources in savannas. Ecosystems, 3: 82-97.

MMAMRM, 2008. EL programa S.A.H.I.: Descripción y Funcionalidad. El presente y el futuro del Sistema. Ministerio de Medio Ambiente y Medio Rural y Marino, 31 pp., Madrid.

OLIVARES GUILLEM. A., 2004. Modelización hidrológica pseudodistribuida del Barranc del Carraixet: aplicación al episodio de octubre de 2000. Cuadernos de Geografía, 76: 155-182.

SHA, S.M.S.; O'CONNELL, P.E. \& HOSKING, J.R.M., 1996. Modelling the effects of spatial variability in rainfall on catchment response. 2. Experiments with distributed and lumped models. Journal of Hydrology, 175: 89-111. 
J. A. PASCUAL AGUILAR, J. L. RUBIO, V. ANDREU, J. CAMPO, O. GONZÁLEZ \& E. GIMENO

YAIR, A. \& RAZ-YASSIF, N., 2004. Hydrological processes in a small arid catchment: scale effects of rainfall and slope length. Geomorphology, 61: 155-169.

YILDIZ, O. \& BARROS, A. P., 2009. Evaluating spatial variability and scale effects on hydrologic processes in a midsize river basin. Scientific Research and Essay, 4(4): 217-225. 